\title{
FOTOGEOLOGÍA DE LOS COMPLEJOS VOLCÁNICOS EL HOYO Y ASOSOSCA (NICARAGUA)
}

\author{
Elena Badilla, Ignacio Chaves, Lepolt Linkimer, Héctor Zúñiga \& \\ Guillermo E. Alvarado \\ Escuela Centroamericana de Geología, Universidad de Costa Rica, \\ Apdo. 214, 2060 Costa Rica
}

(Recibido 29/7/1999; Aceptado 3/3/2000)

\begin{abstract}
Volcanic structures associated with a N-S cortical fissure were distinguished between Puerto Momotombo and Malpaisillo. The Volcanic Complex El Hoyo is the result of at least three different eruption cycles. Development and collapse of the caldera of Picacho volcano, construction of Paleo Hoyo volcano and El Hoyo volcano, which has been active in 1528, 1952 and 1954. Less complicate structures correspond with the Asososca and The Piles volcanoes, which together with the El Hoyo Complex generated a series of lava flows predominantly toward north and northeast producing irregular topography. The maares Malpaisillo and Asososca Lagoon are the result of freatomagmatic events, in association with a cortical fissure. The area has a high risk because the presence of NNW normal faults and volcanoes, it would be necessary to prevent and mitigate damages in towns like, Puerto Momotombo, the America and Malpaisillo.
\end{abstract}

\begin{abstract}
RESUMEN: Un conjunto de geoformas volcánicas, asociadas con fisuras corticales de rumbo N-S, se distinguen entre Puerto Momotombo y Malpaisillo.El Complejo Volcánico El Hoyo es el resultado de una secuencia de por lo menos tres etapas: desarrollo y colapso caldérico del volcán El Picacho, edificación del volcán Paleo-Hoyo y la actividad del volcán El Hoyo, activo en 1528, 1952 y 1954. Otras estructuras más simples son los maares Laguna Asososca y Malpaisillo y los estratovolcanes Asososca y Las Pilas. Este último, junto con los volcanes del Complejo El Hoyo, genera una serie de coladas orientadas predominantemente hacia el norte y el noreste. Las unidades son afectadas por posibles fallas normales, y activas, con una tendencia general NNW.

La región posee un peligro volcánico elevado relacionado con la emisión de coladas de lava, flujos piroclásticos, tefras de caída y lahares. Sin embargo, la expulsión de ceniza es la actividad que hace más vulnerables a las comunidades cercanas. El poblado de Malpaisillo presenta un peligro volcánico mayor, ligado a una eventual actividad freatomagmática producida a partir de la posible fisura cortical sobre la que se encuentra situada esta comunidad.
\end{abstract}

\section{INTRODUCCIÓN}

La configuración geológica de Nicaragua se originó en el Paleozoico y culminó con una intensa actividad a principios del Cuaternario (Weyl, 1980; Giesecke, 1989). Las rocas volcánicas del Terciario y aún del Cretácico que conforman la parte central de Nicaragua, están ordenadas en por lo menos doce paleoarcos volcánicos. La actividad volcánica durante el Terciario ha migrado de este a oeste y existe cierta relación entre las estructuras de caldera y las zonas de falla principales. La disposición de los paleoarcos volcánicos controla en gran parte la geomorfología del área central de $\mathrm{Ni}$ caragua (Valrey, 1988). Dengo et al. (1970) y Alvarado et al. (1986) describen que en Centroamérica los grupos individuales de conos volcánicos están localizados a lo largo de fallas N-S.

Las estructuras relacionadas con la actividad neovolcánica más reciente en el territorio nicaragüense, incluyen por lo menos diez estratovolcanes, nueve de ellos activos desde la conquista, complejos maáricos, caldéricos y conos piroclásticos (Simkin \& Siebert, 1994). Una de estas estructuras es el complejo volcánico conformado por los volcanes El Hoyo, Las Pilas, Asososca, El Rota y Cerro Negro. 
Martínez \& Viramonte (1971, en Weyl, 1980) elaboraron un mapa geológico del complejo volcánico El Hoyo en el que, por su escala, no se pueden discernir los detalles geológicos.

Walker (1983) señala que el volcán Las Pilas-El Hoyo posee erupciones históricas. Simkin \& Siebert (1994) reportan que el volcán El Hoyo es un volcán complejo con erupciones en 1528, 1952 y 1954, las cuales fueron principalmente centrales, explosivas y freáticas. Según Weyl (1980), El Hoyo desarrolló actividad fumarólica en 1977. Van Wyk de Vries (1993) indica que en el macizo El Hoyo han habido dos focos volcánicos activos en tiempos recientes. Hay un eje central con tendencia hacia el este, formado por numerosos cráteres y muchos conos subsidiarios en lineamientos con tendencia norte. $\mathrm{La}$ actividad lateral ha producido conos de cenizas y maares, mientras que la actividad del foco central ha sido casi exclusivamente efusiva; los únicos depósitos piroclásticos han sido aglomerados y delgados niveles de escorias.
El Cerro Negro es el volcán más reciente de Nicaragua, surgió en 1850 y a partir de entonces han ocurrido una serie de erupciones: 1850, 1867, 1899, 1914, 1919, 1923, 1929, 1947-50, 1954, 1957, 1960-64, 1968-69, 1971, 1992 (Simkin \& Siebert, 1994), 1995 y 1999, siendo la de 1968 una de las más violentas (Giesecke, 1989).

Este estudio tiene como objetivo describir las estructuras que componen dicho complejo volcánico, con el fin de establecer las relaciones volcanomorfológicas existentes, determinar una posible secuencia de eventos e identificar en forma preliminar los peligros volcánicos que se puedan generar. Para esto se realizó un análisis detallado de un área de $130 \mathrm{~km}^{2}$ ubicada entre Puerto Momotombo y Malpaisillo (Fig. 1), Departamento de León, Nicaragua, a partir de seis fotografías aéreas (R11, línea 34, fotografías 2568 , 2569, 2570 y R04, línea 33, fotografías 889, 890, 891) escala 1:40 000. Además, se llevó a cabo una revisión bibliográfica y una interpretación a partir de los datos obtenidos.

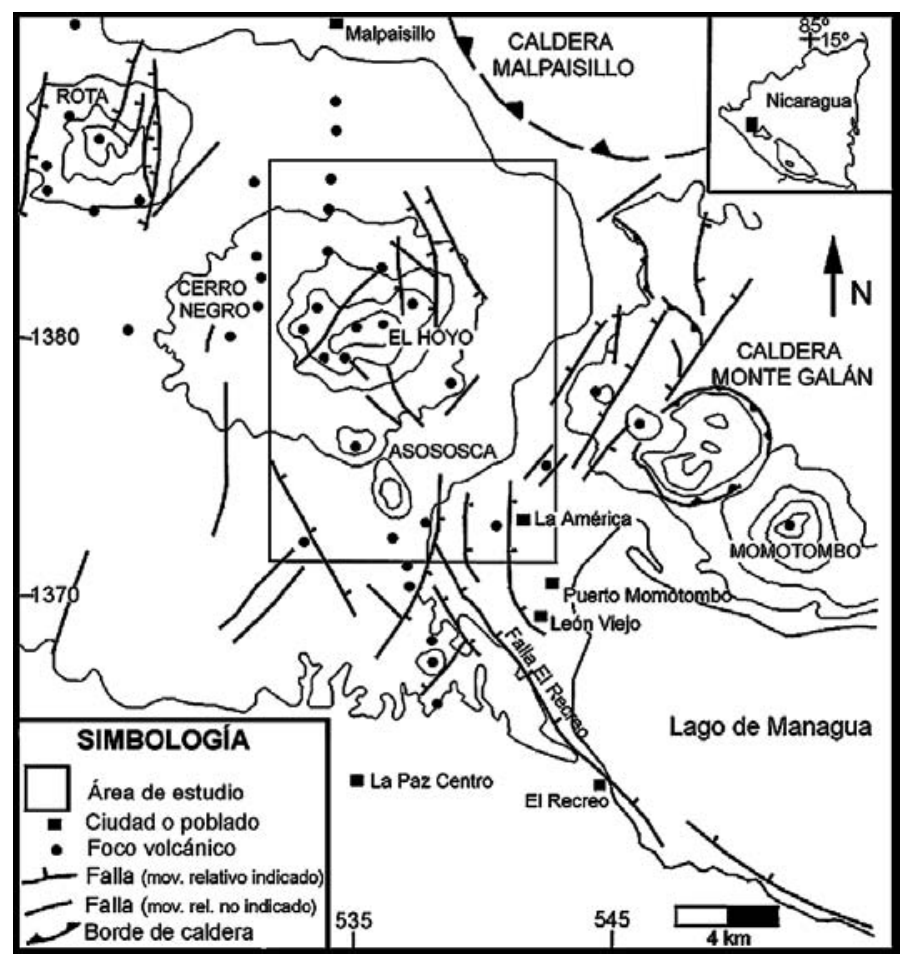

Fig. 1: Mapa de ubicación y estructural del área de estudio (modificado de Van Wyk de Vries, 1993). 


\section{UNIDADES GEOMORFOLÓGICAS}

Las siguientes unidades fueron definidas con base en las fotografías aéreas mencionadas anteriormente. En la figura 2 se muestra la distribución espacial de estas unidades.

\section{Relictos Volcánicos}

Esta unidad comprende los cerros Cabeza de Vaca (666 m.s.n.m.), Tacanistes (512 m s.n.m) y otros con alturas de 195, 302 y 780. Las pendientes máximas alcanzan los $20^{\circ}$. Sus bases

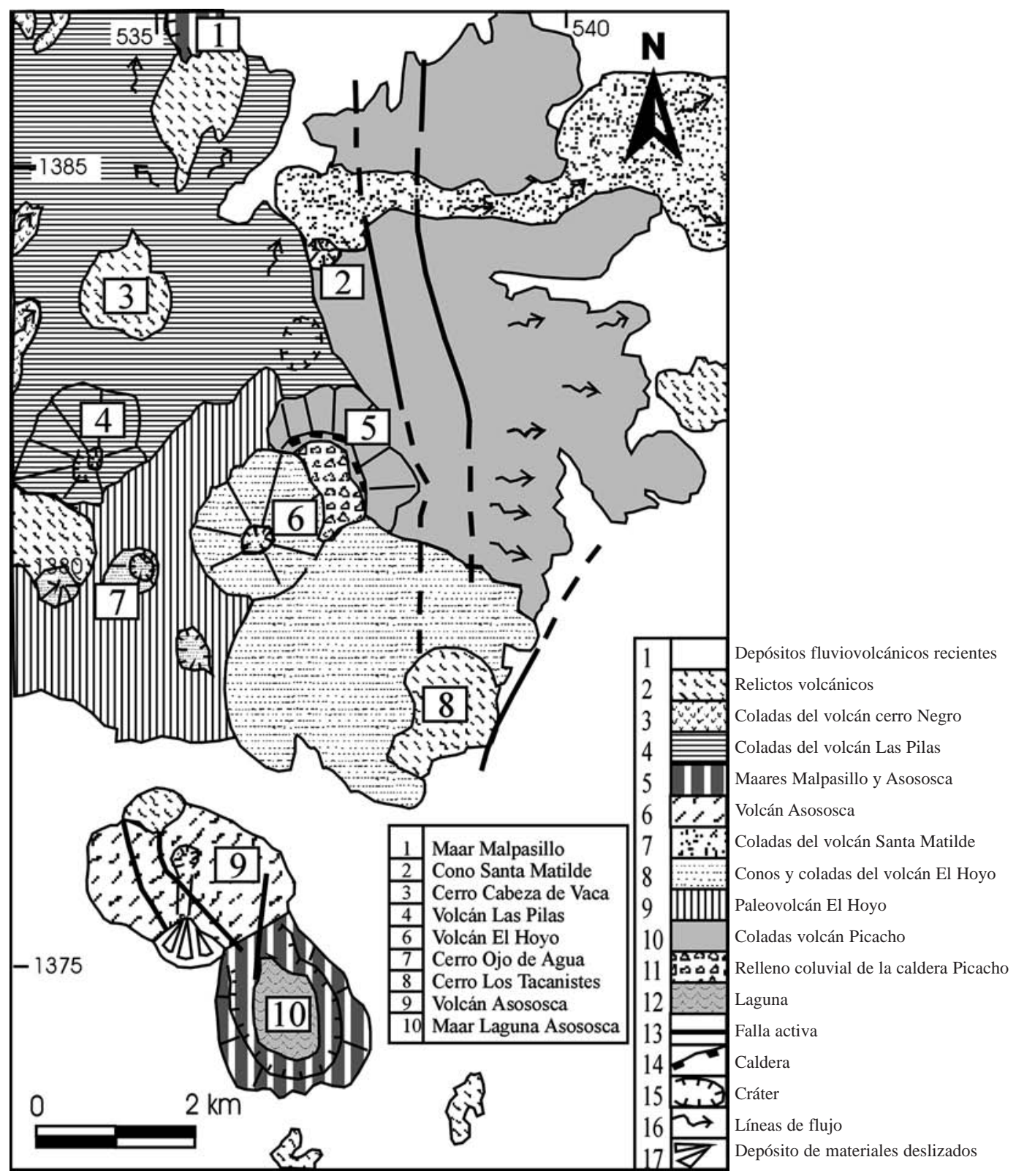

Fig. 2: Mapa fotogeológico de los complejos estratovolcánicos El Hoyo y Asososca. 
son redondeadas con un diámetro máximo de 1200 m; el patrón de drenaje es radial y generalmente se presentan rodeados por coladas que provienen de otros centros de emisión cercanos. Se desconoce si se trata de antiguos focos volcánicos erosionados o son restos de coladas y/o de tobas soldadas.

\section{Complejo Volcánico El Hoyo}

Abarca aproximadamente $50 \mathrm{~km}^{2}$ e incluye una serie de geoformas correspondientes con diferentes eventos de la evolución volcánica de la región. Se distinguieron las siguientes estructuras:

\section{Caldera El Picacho}

Corresponde con los restos de un paleovolcán colapsado, representado por una estructura semicircular que posee un diámetro de $1,5 \mathrm{~km}$, con un escarpe de unos $80 \mathrm{~m}$ de alto y laderas exteriores con pendientes que alcanzan los $14^{\circ}$. Esta caldera se encuentra rodeada por una serie de coladas de lava afectadas por fallas N-S y desarrolladas posiblemente en el antiguo volcán que dio origen a esta estructura.

Con base en el grado de erosión superficial se distinguieron dos grandes campos de coladas. El primero tiene una longitud de aproximadamente $2 \mathrm{~km}$ y una dirección predominante hacia el SE; su patrón de drenaje es paralelo y está muy afectado por la erosión hídrica que genera cárcavas. El segundo posee una longitud de $4 \mathrm{~km}$ y presenta lóbulos frontales que corresponden con al menos tres flujos superpuestos que están totalmente cubiertos de bosque. Ambos campos de coladas presentan pendientes poco uniformes y superficies cubiertas de crestas de presión.

En el interior de esta caldera se presenta una zona plana cubierta de vegetación, que corresponde con un relleno parcial constituido posiblemente por materiales volcaniclásticos (coluvios o depósitos fluviolacustres) provenientes del volcán Paleo-Hoyo, del volcán El Hoyo o de la erosión de la caldera misma.

\section{Volcán Paleo-Hoyo}

Con este término se define la estructura volcánica previa al actual volcán El Hoyo. Posee un diámetro de $4 \mathrm{~km}$, con una pendiente uniforme de $14^{\circ}$, un patrón de drenaje radial y zonas con o sin vegetación, quizás producto de lluvia ácida y/o cenizas provenientes del actual volcán El Hoyo.

\section{Volcán El Hoyo}

Estructura de base circular de $2 \mathrm{~km}$ de diámetro con una altura de $1088 \mathrm{~m}$ y pendientes uniformes de $20^{\circ}$ compuestas por una sucesión de coladas y piroclastos intercalados. Posee tres cráteres de paredes verticales de 400, 80 y $200 \mathrm{~m}$ de diámetro, respectivamente, alineados en dirección N-S. El mayor de estos, se encuentra en la cima, tiene forma de embudo escalonado y una profundidad de aproximadamente $500 \mathrm{~m}$, con fracturas transversales en el borde.

Asociado con este volcán se encuentra hacia el sureste un campo de coladas de lava que presenta una superficie poco erosionada de $3 \mathrm{~km}$ de longitud, con relieve irregular. Su espesor es de unos $40 \mathrm{~m}$, abarca un área de 7,6 km² y posee un volumen estimado de $0,3 \mathrm{~km}^{3}$. De acuerdo con Van Wyk de Vries (1993), las características de estas coladas son su gran extensión y los túneles de lava y superficies pahoehoe. Aunque este autor establece que el volcán El Hoyo es un escudo volcánico, en este estudio se le considera como un volcán achatado, dadas sus dimensiones pequeñas.

Relacionado con el complejo El Hoyo existe un pequeño cono excéntrico, llamado Santa Matilde, de $381 \mathrm{~m}$ de altura, $400 \mathrm{~m}$ de diámetro en la base y laderas uniformes. Tiene un cráter circular en la cima, de 80 m de diámetro, que se encuentra desportillado hacia el norte. De él proviene una colada de lava de $7 \mathrm{~km}$ de largo, que ocupa un área de $6,7 \mathrm{~km}^{2}$ y un volumen de $0,3 \mathrm{~km}^{3}$. Además, dentro de este complejo se incluyen dos cráteres parásitos ubicados al norte y al suroeste del volcán El Hoyo, representados por depresiones de 600 y 500 m de diámetro respectivamente, con formas elipsoidales y profundidades de 
hasta $20 \mathrm{~m}$. Se encuentran erosionados y uno de ellos posee un patrón de drenaje paralelo que provoca un desgaste mayor hacia el norte.

\section{Volcán Cerro Ojo de Agua}

Incluye tanto al volcán Ojo de Agua (813 m.s.n.m.) como a un pequeño cono que se ubica hacia el oeste (720 m de elevación). El primero posee $600 \mathrm{~m}$ de diámetro en su base y un cráter de $200 \mathrm{~m}$ de diámetro y $80 \mathrm{~m}$ de profundidad. El segundo tiene un cráter de $100 \mathrm{~m}$ de diámetro desportillado hacia el NE. Ambos sobresalen del terreno circundante con un máximo de $100 \mathrm{~m}$, presentan una pendiente uniforme de $20^{\circ}$ y no poseen vegetación. Se interpretan como conos parásitos del volcán El Hoyo.

\section{Maares}

Se distinguieron dos maares, lagos cratéricos cuyo piso se ubica por debajo del nivel del terreno circundante:

\section{Maar Malpaisillo}

Depresión de $700 \mathrm{~m}$ de ancho y $1000 \mathrm{~m}$ de longitud $\left(0,7 \mathrm{~km}^{2}\right)$ con una profundidad de unos $100 \mathrm{~m}$ y un fondo llano ocupado en parte por una laguna. Sus paredes internas son verticales, sin signos de erosión, y se encuentran rodeadas y parcialmente cubiertas de coladas de lava pertenecientes al volcán Las Pilas. Se alinea en sentido N-S con otros maares menores.

\section{Maar Laguna Asososca}

Depresión ovalada de 1500 m de longitud y 1000 m de ancho, localizada al SE del volcán Asososca. Está rodeado por un anillo de cenizas, de posible origen freatomagmático, que tiene una altura de $55 \mathrm{~m}$ sobre el terreno circundante y de $160 \mathrm{~m}$ sobre el nivel del lago que ocupa el fondo de la depresión. El borde oeste del anillo posee una ladera externa con inclinación de $10^{\circ}$ y una interna con pendiente de $25^{\circ}$. En el lado este, las laderas externa e interna tienen una inclinación de $8^{\circ}$ y $38^{\circ}$, respectivamente.
En el sector norte del maar se distinguen dos depresiones con una orientación SW-NE y un diámetro cercano a $200 \mathrm{~m}$, las cuales fueron causadas probablemente por explosiones volcánicas.

De acuerdo con los datos aportados por Weyl (1980), se establece que los materiales de este maar son andesitas basálticas y basaltos con olivino. Sin embargo, se requiere una revisión geoquímica de las tefras juveniles para examinar si existen dos magmas envueltos durante la formación del maar o si hay xenolitos.

\section{Volcán Las Pilas}

Consiste de una estructura de forma cónica, de $1001 \mathrm{~m}$ de elevación, que se eleva hasta $300 \mathrm{~m}$ sobre la superficie circundante; posee una base casi circular de $1,5 \mathrm{~km}$ de diámetro y presenta en la cima una estructura de colapso, la cual tiene en su interior un cráter de $250 \mathrm{~m}$ de diámetro y $20 \mathrm{~m}$ de profundidad.

El campo de coladas de este volcán corresponde con una superficie irregular con morfología que denota claramente un flujo hacia el norte, totalmente cubierto de vegetación. Por el grado de desarrollo de la vegetación, se interpreta la existencia de 3 flujos con un espesor máximo de $60 \mathrm{~m}$, que cubren un área de $16,4 \mathrm{~km}^{2}$ y ocupan un volumen estimado de $0,96 \mathrm{~km}^{3}$.

\section{Cerro Asososca}

Edificio volcánico con laderas de $22^{\circ}$ de inclinación y una altitud de $818 \mathrm{~m}$. Se eleva unos $620 \mathrm{~m}$ sobre el nivel del terreno circundante y posee una base circular de 2,4 km. Hacia el sur, se puede observar un abanico formado por un deslizamiento o flujo de escombros originado desde su cima. En el flanco NW existe una estructura posiblemente más antigua debido a un mayor desarrollo del patrón de drenaje.

\section{Volcán Cerro Negro}

En la parte noreste del área de estudio existen algunas coladas de lavas recientes, notoriamente oscuras en las fotografías aéreas, casi llanas y con líneas de flujo, provenientes 
del volcán Cerro Negro, el cual se encuentra fuera del área de estudio. Este volcán está considerado dentro de los más activos de América Central y presenta un período eruptivo cada 2 a 32 años, con un promedio de una erupción cada 9 años.

\section{Llanura fluviovolcánica}

Zona casi plana con una pendiente uniforme de $3^{\circ}$ y un área de más de $40 \mathrm{~km}^{2}$. El patrón de drenaje es paralelo y origina cárcavas en algunos sectores. Estas áreas son utilizadas para actividades agrícolas.

\section{MARCO ESTRUCTURAL}

Las estructuras presentes en el área de estudio se destacan en las figuras 1 y 2 y a continuación se hará una descripción de las principales:

\section{Alineamientos de volcanes}

Los volcanes principales y las estructuras volcánicas menores se presentan alineados a lo largo de lo que se interpretó como fisuras corticales profundas. Estas son:

a) Alineamiento de la cadena neovolcánica de Nicaragua con rumbo $\mathrm{N} 63^{\circ} \mathrm{W}$ (p.e. volcanes El Hoyo, Las Pilas y Cerro Negro) y alineamiento con rumbo $\mathrm{N} 54^{\circ} \mathrm{W}$ de los volcanes Momotombo, Montoso, Los Palomos, Colorado y la Caldera Monte Galán. Ambos ejes presentan una aparente separación lateral siniestral de unos $2 \mathrm{~km}$.

b) Alineamientos transversales al eje neovolcánico con rumbo aproximado $\mathrm{N}-\mathrm{S}$, de los cuales se distinguen, al menos, los volcanes Cerro Negro y La Mula; Asososca y Ojo de Agua, cerro Cabeza de Vaca y maar Malpaisillo y, maar Laguna Asososca, volcán El Hoyo y cono Santa Matilde.

\section{Fallas}

Las geoformas volcánicas observadas son afectadas por una serie de fallas, posiblemente normales, que se describen a continuación:

a) Dos lineamientos ubicados al este del volcán El Hoyo con rumbo $\mathrm{N} 5^{\circ} \mathrm{W}$ y longitudes de 8 y $7 \mathrm{~km}$, respectivamente. Estos fueron interpretados como fallas, sin embargo la presencia de un relieve positivo puede ser indicativo de diques erosionados, por lo que se recomienda su comprobación de campo.

b) Falla posiblemente activa, con rumbo $\mathrm{N} 30^{\circ} \mathrm{E}$ y $3 \mathrm{~km}$ de longitud, que afecta los depósitos fluviovolcánicos al este del volcán Las Pilas.

c) Dos fallas normales con rumbo aproximado $\mathrm{N} 45^{\circ} \mathrm{W}$, con longitudes de 2 y $1 \mathrm{~km}$, ubicadas en la ladera oeste del volcán Asososca y una falla con rumbo $\mathrm{N}^{\circ} \mathrm{E}$ y 1 $\mathrm{km}$ de longitud, en el flanco este del mismo volcán.

d) Falla con rumbo $\mathrm{N} 20^{\circ} \mathrm{W}$ y $2 \mathrm{~km}$ de longitud que afecta las coladas de la actual caldera El Picacho.

\section{PELIGRO VOLCÁNICO PRELIMINAR}

El pueblo de Malpaisillo puede verse afectado por actividad freatomagmática, con la consecuente formación de nuevos maares, relacionados con la presencia de una serie de fisuras corticales paralelas con rumbo N-S. La emisión de coladas de lava, flujos piroclásticos y tefras, asociada con una eventual reactivación de los volcanes El Hoyo y Cerro Negro, de los cuales se conoce actividad histórica, es un peligro para las comunidades aledañas a estos volcanes. La expulsión de cenizas ocasionaría daños a los poblados ubicados al SW del complejo volcánico El Hoyo, debido a la dirección de los vientos. A pesar de esto, se carece de suficiente información para especular sobre la prontitud de estos eventos. 
Los lahares también representan un peligro para las poblaciones que se ubican en las zonas planas al pie de los volcanes y cerca de los ríos principales. Un caso particular es el abanico ubicado al pie del flanco sur del volcán Asososca.

Pueden presentarse movimientos sísmicos asociados a fallas neotectónicas, como las localizadas en el volcán Asososca. Carr \& Stoiber (1977), White (1991) y White \& Harlow (1993) reportan, en los sectores aledaños a los volcanes estudiados, la ocurrencia de microtemblores y de terremotos superficiales, históricamente dañinos, con magnitudes intermedias entre 5 y 6,5 . La existencia de estas fallas activas, junto con la sismicidad intraplaca somera, plantea el análisis de la peligrosidad sísmica para estudios posteriores.

\section{CONCLUSIONES}

Con base en el análisis de sobreposición de estructuras volcánicas y grado de erosión, se pudo establecer una secuencia de eventos volcano-estructurales y morfoestratigráficos.

El complejo El Hoyo se inicia con la formación del volcán El Picacho, el cual derrama una serie de coladas de unos $4 \mathrm{~km}$ de longitud durante sus diferentes períodos de actividad, sufriendo posteriormente un colapso que forma una caldera de por lo menos 1,5 km de diámetro. Se desconoce si existen depósitos ignimbríticos asociados con dicho colapso. Un nuevo volcán se forma en el interior de la caldera (volcán Paleo-Hoyo) y posteriormente colapsa o se erosiona, rellenando sectores en el interior de la caldera.

Después de un lapso prolongado de tiempo, un nuevo período de actividad origina la aparición de una serie de pequeños volcanes, depresiones y maares en las inmediaciones de la caldera. En este mismo período se pudieron generar los volcanes Las Pilas, El Hoyo y Asososca, que derramaron coladas de lava y emitieron flujos piroclásticos. La erosión de estas estructuras volcánicas provoca extensas áreas de depositación con una morfología llana y hoy día ampliamente utilizadas con fines agrícolas. El fallamiento es intenso y ocurre a lo largo de fracturas N-S y NW-SE.
Las últimas manifestaciones del vulcanismo en tiempos recientes están representadas por las erupciones de los volcanes El Hoyo y el Cerro Negro. Las erupciones del primero son principalmente explosivas, mientras que las del segundo son del tipo estromboliano y hawaiano con coladas de lava.

Los volcanes principales y las estructuras volcánicas se presentan alineados, preferencialmente a lo largo de lo que se interpretó como fisuras corticales profundas con rumbo N-S y NW-SE.

\section{AGRADECIMIENTOS}

Este trabajo fue realizado como parte del curso de Vulcanología (G-5120) del primer semestre de 1999, de la Escuela Centroamericana de Geología (UCR), como un aporte al Instituto Nacional de Estudios Territoriales (INETER), dentro del marco de evaluación de las amenazas volcánicas y gracias a la colaboración del material fotográfico y de mapas cedido por la Ing. Marta Navarro.

\section{REFERENCIAS}

ALVARADO, G.E., BARQUERO, R., BOSCHINI, I., CHIESA, S. \& CARR, M., 1986: Relación entre la neotectónica y el vulcanismo en Costa Rica. - Bogotá, Simposio internacional sobre neotectónica y riesgos volcánicos, 19 págs.

CARR, M.J. \& STOIBER, R.E., 1977: Geologic setting of some destructive earthquakes in Central America. - Geol. Soc. Amer. Bull. 88: 151-156.

DENGO, G., BOHNENBERGER, O. \& BONIS, S., 1970: Tectonics and volcanism along the Pacific marginal zone of Central America. Geol. Rundsch. 59: 1215-1232.

GIESECKE, A.(coord.), 1989: Riesgo volcánico, evaluación y mitigación en América Latina: aspectos sociales, institucionales y científicos. 
- 298 págs. Centro Regional de sismología para América del Sur, Lima.

SIMKIN, T. \& SIEBERT, L., 1994: Volcanoes of the world. - 349 págs. Arizona, Geoscience Press, Inc., Smithsonian Institution.

VALREY, G., 1988: Desplazamiento de actividades volcánicas y su relación entre paleo arcos antiguos y depósitos minerales en Nicaragua. - Quito, Memorias VI Curso Internacional de Metalogenia, Universidad Central del Ecuador, Facultad de Ingeniería en Geología, Minas y Petróleo: 241 - 251.

VAN WYK DE VRIES, B., 1993: Tectonics and magma evolution of Nicaraguan volcanic systems. - 328 págs. The Open University, London [tesis $\mathrm{PhD}$.]
WALKER, J.A., 1983: Volcanic rocks from the Nejapa and Granada cinder cone alignments, Nicaragua, Central America. - J. Petrology, 25(2): 299 - 342.

WEYL, R., 1980: Geology of Central America. 371 págs. Springer Verlag, Berlin.

WHITE, R.A., 1991: Tectonic implications of upper-crustal seismicity in Central America. -En: Slemmons, D.B., Zoback, E.R. \& Blackwell, D.D. (eds.): Neotectonics of North America. Boulder, Colorado, Geol. Soc. Amer. Decade Map Volume 1: 323 - 337.

WHITE, R.A. \& HARLOW, D., 1993: Destructive upper-crustal earthquakes of Central America since 1900. - Bull. Seismol. Soc. Amer. 83(4): 1115 - 1142. 